\title{
Identificación de ectoparásitos en Oso melero (Tamandua tetradactyla), Estado Lara, Venezuela
}

\section{Identification of ectoparasites from an Lesser anteater (Tamandua tetradactyla), Lara State, Venezuela}

\author{
María Forlano R, ${ }^{1 *}$ Ph.D, Franklin Mujica $L_{,}{ }^{1} \mathrm{Ph} . \mathrm{D}$, Johanmary Gallardo $\mathrm{Y}_{,}{ }^{2}$ M.Sc, \\ José Rodríguez $L_{1}^{2}$ M.Sc.
}

\begin{abstract}
${ }^{1}$ Universidad Centroccidental "Lisandro Alvarado", Decanato de Ciencias Veterinarias, Unidad de Investigación en Parasitología Veterinaria, Barquisimeto. Estado Lara, Venezuela. ${ }^{2}$ Universidad Centroccidental "Lisandro Alvarado", Decanato de Ciencias Veterinarias, Área de Anatomía Macroscópica Veterinaria, Barquisimeto. Estado Lara, Venezuela. *Correspondencia: mforlano@ucla. edu.ve
\end{abstract}

Recibido: Noviembre de 2012; Aceptado: Marzo de 2013.

\section{RESUMEN}

Objetivo. Identificar especies de ectoparásitos encontrados parasitando naturalmente un Oso melero, el cual fue encontrado muriendo en una vía cercana al pueblo de Bobare, estado Lara, Venezuela. Materiales y métodos. Al ingresar el animal a la sala de anatomía del DCV-UCLA para ser sometido a la técnica de taxidermia, se procedió a revisar a través de la palpación toda la superficie corporal del Oso, con la finalidad de tomar muestras de ectoparásitos presentes en el hospedador; los especímenes encontrados fueron colocados en un envase de vidrio conservados en alcohol isopropílico y posteriormente fueron llevados al Laboratorio de Parasitología Veterinaria del DCV-UCLA para su identificación taxonómica. Los ectoparásitos fueron observados en lupa estereoscópica y clasificados con ayuda de claves taxonómicas. Resultados. Se identificaron dos especies de artrópodos infestando naturalmente el Oso Melero, de las cuales se encuentra una garrapata macho clasificada como Amblyomma nodosum; y una pulga hembra identificada como Pulex irritans. Conclusiones. Estos artrópodos se han reportado parasitando naturalmente algunas especies de mamíferos, dentro de los cuales el Oso Melero se ha visto afectado por la acción de estos ectoparásitos; en Venezuela es el primer reporte de Pulex irritans afectando esta especie animal.

Palabras clave: Ectoparásitos, garrapatas, pulgas, Oso Melero, Tamandua tetradactyla (Fuente: $D e C S, C A B)$. 


\begin{abstract}
Objective. Identify species of ectoparasites found naturally parasitizing an anteater, which was found dying on the roadside close to Bobare Village, State of Lara, Venezuela. Materials and methods. A dead anteater was brought to the anatomy Lab of the Decanato de Ciencias Veterinarias (DCV), Universidad Centroccidental "Lisandro Alvarado" (UCLA) for taxidermic purposes. First, all the skin and hairy areas of the animal were manually checked looking for ectoparasites; secondly, all detected specimens were collected, and placed in vials containing a solution of isopropilic alcohol $70^{\circ}$. Following this procedure, the vials were taken to the Veterinary Parasitology Laboratory at DCV-UCLA to accomplish the taxonomic identification under a stereoscopic microscope, and with taxonomic keys. Results. Two specimens of arthropods were found, the first one was a male tick identified as Amblyomma nodosum, and the other one was a female flea of the genera Pulex irritans Conclusions. These ectoparasites have been reported as natural parasites of other mammals, and among them the anteaters have been often found parasitized by these arthropods. In Venezuela this is the first case of a Pulex irritans infecting Tamandua tetradactyla.
\end{abstract}

Key words: ticks, fleas, anteater, ectoparasites, Tamandua tetradactyla. (Sources: DeCS, CAB)

\title{
INTRODUCCIÓN
}

El Oso Melero común, Tamandua tetradactyla es un mamífero sudamericano que puede ser observado desde Venezuela hasta el noreste de Argentina, sur de Brasil y Norte de Uruguay (1). En Venezuela se ubica en una gran cantidad de ambientes; desde sabanas arboladas en los llanos entre arbustos, zonas bajas áridas y calurosas, montañosas y húmedas, altas y frías, hasta bosques enanos sobre los Tepuyes del escudo guayanés. También se conoce como oso colmenero y tamanduá (en Colombia, Ecuador y Perú); tamanduá-colete, y tamandua-mirim (en Brasil) (1)

Los Osos Meleros pertenecen a la familia Myrmecophagidae, dentro del orden Xenarthra; su nombre científico es $T$. tetradactyla. Vive en selvas y bordes de sabanas de climas medios y cálidos hasta los $2.000 \mathrm{~m}$ de altura $(1,2)$. A pesar de ser una especie tolerante a una gran cantidad de ambientes y con una amplia distribución en el país, su condición de supervivencia es vulnerable debido a la reducción de sus poblaciones; los cazadores los eliminan porque matan o hieren a sus perros y frecuentemente son atropellados por vehículos en las carreteras (2). El Oso melero está incluido en el Apéndice II del CITES (Convención Internacional del Tráfico de Especies en Peligro, por sus siglas en inglés), el cual se encuentra reservado para las especies que aún en peligro, su población no está al borde de la extinción (3).

Como cualquier especie de hábitats abiertos, estos hospedadores resultan susceptibles a diversos agentes patógenos, entre ellos ectoparásitos como los insectos y las garrapatas, que son reconocidos por parasitar vertebrados domésticos, silvestres y al hombre, lo cual puede resultar en problemas sanitarios para sus hospedadores $(4,5)$. Las garrapatas son ectoparásitos que están adaptados a vivir en una gran variedad de hospederos vertebrados tales como: anfibios, reptiles, aves y mamíferos (5).

En diversos países han reportado especies de garrapatas duras infestando a $T$. tetradactyla, como las diagnosticadas en Perú donde se identificaron especies de Amblyomma calcaratum, estos autores mencionan las relaciones filogenéticas de esta especie con $A$. nodosum y de su importancia médica (6). En Brasil se han identificado especies de Amblyomma sp. $A$. cajennense, $A$. parvum y $A$. nodosum haciendo énfasis que esta última especie es un parásito específico de Tamandúa $(7,8)$. En México se han diagnosticado en Tamandua mexicana la especie de Amblyomma calcaratum (9). En Colombia se identificó Amblyomma sp. en T. tetradactyla (10).

A. nodosum se ha diagnosticando parasitando Oso Melero en localidades de Chaco y Formosa de Argentina (11), así como en Bolivia, Guatemala, Costa Rica, Nicaragua, Panamá, Trinidad y Tobago parasitando $T$. tetradactyla (12). En Venezuela A. nodosum fue reportada en especies de Xenarthra, existiendo un vacío de reportes desde la fecha, aunque algunos autores han observado que $A$. calcaratum es una garrapata común en estos mamíferos (Tamandua, Myrmecophaga), reportan que frecuentemente se les puede encontrar junto con adultos de $A$. nodosum y que el diagnostico diferencial de ambas especies es 
difícil de realizar, lo que conlleva muchas veces a inferir en diagnósticos incorrectos (11).

En relación a la presencia de Siphonaptera parasitando esta especie animal se han reportado pocos hallazgos, en Venezuela no se tienen reportes que indiquen la infestación de estos insectos en Oso Melero; sin embargo, se ha reportado en Brasil Pulex irritans parasitando naturalmente a tamandúa (4), así como en el centro de los Estados Unidos las infestaciones fuertes en Myrmecophaga tridactyla con una especie de pulgas la cual diagnosticaron como Pulex simulans. Este insecto se encuentra comúnmente parasitando otras especies como Didelphis virginiana y los Procyon lotor (13). El objetivo de este estudio fue identificar morfológica y taxonómicamente los ectoparásitos encontrados infestando naturalmente a un Oso Melero encontrado muriendo en una localidad del estado Lara, Venezuela.

\section{MATERIALES Y MÉTODOS}

Sitio de estudio. Esta investigación fue realizada en la unidad de investigación de Parasitología Veterinaria del Decanato de Ciencias Veterinarias de la Universidad Centroccidental "Lisandro Alvarado" (UCLA) en el estado Lara-Venezuela.

Recolección de la muestra. Las muestras fueron extraídas manualmente de un Oso Melero común (Tamandua tetradactyla) que fue encontrado muriendo en una carretera que conduce a la población de Bobare (10015'55,86"LN, 69028'24,50"LO), estado Lara, y que murió al poco tiempo de haber sido auxiliado; razón por la cual fue donado al Área de Anatomía de los Animales Domésticos del Decanato de Ciencias Veterinarias de la UCLA, donde se decidió someterlo a la técnica de taxidermia.

Toma de las muestras. Se procedió a la realización de un examen minucioso de todo el cuerpo del animal, por medio de palpación utilizando guantes. Se encontraron artrópodos aun fijados a la piel del animal como una garrapata y en movimiento sobre la superficie del cuerpo una pulga parasitando al Oso, las cuales fueron extraídas manualmente y conservadas en alcohol isoproplico para su posterior identificación taxonómica y morfológica.
Procesamiento de las muestras. Los ectoparásitos fueron lavados con agua destilada y secados con papel absorbente, posteriormente con la ayuda de la lupa estereoscópica se observaron para ser clasificados utilizando claves taxonómicas de ixodideos (14) y para la clasificación de la pulga se utilizó la clave de sifonápteros de Brasil (4). Los resultados de esta investigación se analizaron con estadísticas descriptivas.

\section{RESULTADOS}

Se identificaron dos ectoparásitos infestando naturalmente el Oso Melero (Tamandua tetradactyla); se identificó una especie de insecto por sus características morfológicas y taxonómicas que pertenece a la familia Pulicidae, género Pulex irritans (Figura 1) es una pulga hembra que presenta tres cerdas: pre-ocular, genal y una tercera cerda larga en la región post-antenal, morfológicamente la espermateca presenta cuerpo arredondeado y el apéndice curvo.

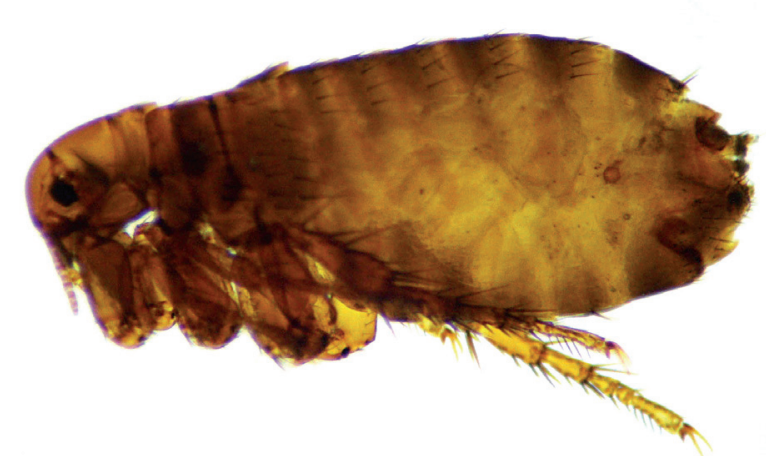

Figura 1. Hembra adulta de Pulex irritans.

Otro ectoparásito encontrado parasitando naturalmente el Oso fue un especime macho de Ixodidae identificado como $A$. nodosum (Figura 2); por presentar caracteres morfológicos como palpos cortos y nudosos, ornamentaciones en el escudo que se presentan en forma de letra "J" en borde lateral anterior del idiosoma de un color amarillo claro, en la vista ventral se observa en la coxa I la presencia de dos espinas gruesas, siendo la externa ligeramente de menor tamaño que la espina interna. 


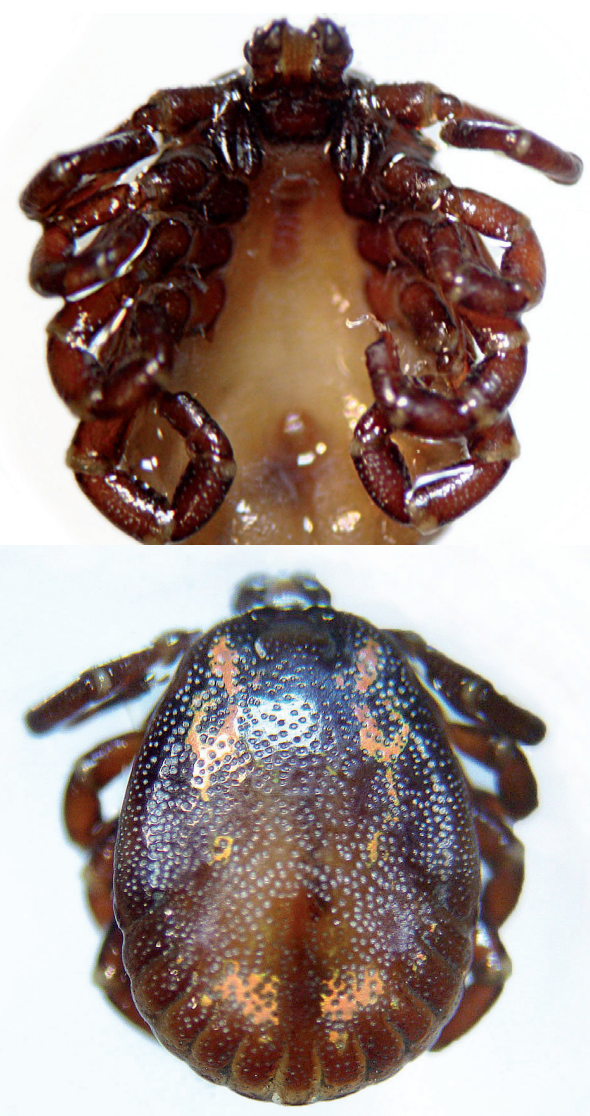

Figura 2. Vista dorsal y ventral de macho Amblyomma nodosum.

\section{DISCUSIÓN}

Los insectos diagnosticados parasitando Tamandua tetradactyla en su hábitat natural son escasos, en Brasil se han reportado Pulex irritans (4) y en el centro de los Estados Unidos se reportó la infestación alta por Pulex simulans en M. tridactyla, en donde los autores reportan que esta especie de insecto se encuentra parasitando comúnmente a esta y otras especies silvestres como $D$. virginiana y los $P$. lotor (13), esta especie sólo se ha reportado en centro de los EEUU y México, la única especie encontrada se diagnosticó morfológicamente a Pulex irritans, que se ha diagnosticado en América del Sur parasitando a $T$. tetradactyla, así como, los animales domésticos y al hombre, este hallazgo puede estar dado por un parasitismo accidental ya que fue observado una única pulga, en un solo animal moribundo (4).

A. nodosum fue reportada parasitando animales silvestres, en Venezuela fue reportada a finales de los años noventa y ahora se reporta afectando al Oso Melero. Similares reportes se han realizado en diferentes países de América Latina con una prevalencia alta del género Amblyomma como principal garrapata encontrada parasitando a $T$. tetradactyla con una gran variedad de especies, donde $A$. nodosum se ha caracterizado como especie específica de Tamandúa y con una gran importancia médica $(6,7,8,12)$.

Una de las preocupaciones con la identificación y registros de algunas especies de garrapatas en animales silvestres está relacionada con el potencial riesgo de estos artrópodos de transmitir patógenos a otros animales silvestres y domésticos, bien como a los humanos (8); en Venezuela conviven diversas especies de animales silvestres y en los últimos años, se ha observado incremento de poblaciones humanas hacia las regiones donde habitan los animales silvestres.

En conclusión se puede observar que aunque $A$. nodosum se ha diagnosticado con frecuencia en T. tetradactyla en otros países; en Venezuela se tienen pocos reportes de este ectoparásito en especies de vida silvestre. En cuanto al sifonáptero Pulex irritans es el primer reporte de esta especie en $T$. tetradactyla en esta región.

\section{REFERENCIAS}

1 Oliveira EV, Vilella FS. Xenarthros. In Livro Vermelho da fauna ameaçada de extinção no Rio Grande do Sul. Porto Alegre, Brasil: EDIPUCRS; 2003.
2. Alves CA, Hobus Q, Bager A. Mammalia, Pilosa, Myrmecophagidae, Tamandua tetradactyla (Linnaeus, 1758): Distribution extension. Check Lists 2010; 6:52-53. URL Disponible en: http://www.researchgate. net/publication/236610723_Mammalia_ Pilosa_Myrmecophagidae_Tamandua_ tetradactyla(Linnaeus_1758)_Distribution_ extension. 
3. Sánchez $F$, Sánchez-Palomino $P$, Cadena A. Inventario de mamíferos en un bosque de Los Andes Centrales de Colombia. Mammal survey in a central Andes forest in Colombia. Caldasia 2004; 26:291-309.

4. Linardi $P$, Guimarães L. Siphonapteros do Brasil. São Paulo FAPESP Brasil: Editorial Winnergraph; 2000.

5. Guglielmone A, Nava A. Las garrapatas Argentinas del género Amblyomma (Acari: Ixodidae), distribución y hospedadores. Revista de Investigaciones Agropecuarias (RIA). Argentina: Ediciones Inst $\mathrm{Nac} \mathrm{Tec}$ Agrop (INTA) 2006; 35(3):133-153.

6. Càceres A, Beati L, Keirans J. First evidence of the occurrence of Amblyomma calcaratum Neumann, 1899 in Peru. Rev Peru Biol 2002; 9:116-117.

7. Labruna M, Paula C, Lima T, Sana D. Ticks (Acari: Ixodidae) of wild animals from the Porto-Primavera Hydroeletric Power Station area, Brazil. Mem Inst Oswaldo Cruz. 2002; 97:1133-1136.

8. Martins J, Medri M, Oliveira C, Guglielmone A. Ocorrência de carrapatos em tamanduábandeira (Myrmecophaga tridactyla) e tamanduá-mirim (Tamandua tetradactyla) na região do Pantanal Sul Mato-Grossense, Brasil. Cienc Rural 2004; 34:293-295.
9. Guglielmone A, Pérez T, Cornejo CS. First records of the ticks Amblyomma calcaratum and $A$. pacae (Acari: Ixodidae) parasitizing mammals of México. Rev Mex Biodivers 2006; 77:123-127.

10. Torres-Mejia A, Fuente J. Risks associated with ectoparasites of wild mammals in the Departamento of Quindio, Colombia. Int J Appl Res Vet Med 2006; 4:187-192.

11. Guglielmone A, Estada A, Keirans J, Robbins R. Las Garrapatas (Acari: Ixodida) de la región zoogeográfica neotropical. Buenos Aires. Ediciones Inst Nac Tec Agrop (INTA), 2004.

12 Marinho R, Benedet, R, Mazzolli M, Marques S. Amblyomma nodosum (Acari: Ixodidae) infesting collared anteater (Tamandua tetradactyla, Linnaeus, 1758) in Urupema, Santa Catarina, Brazil. Biocienc 2009; 17:109-110.

13. Mutlow A, Dryden M, Payne P. Flea (Pulex simulans) infestation in captive Giant Anteaters (Myrmecophaga tridactyla). J Zoo Wildl Med 2006; 37:427-429.

14. Barros-Battesti DM, Arzua M, Bechara G.H. Carrapatos de Importância Médico-Veteriária da Região Neotropical: Um guía ilustrado para identificação de espécies. Instituto de Butantan. São Paulo: ICTTD-3; 2006. 